\title{
EXCHANGE COUPLING AND MAGNETIZATION PROFILES OF BINARY AND TERNARY MAGNETIC MULTILAYERS
}

\author{
F. SÜSS, U. KREY* \\ Institut für Physik II der Universität, 93040 Regensburg, Germany \\ AND S. KROMPIEWSKI \\ Institute of Molecular Physics, Polish Academy of Sciences \\ .Smoluchowskiego 17, 60-179 Poznań, Poland \\ Within a spin-polarized LMTO approach in the atomic-sphere approxi- \\ mation we calculate $a b$ initio the magnetic properties of various binary and \\ ternary multilayers composed of $\mathrm{Fe}, \mathrm{Co}, \mathrm{Ni}, \mathrm{Cr}, \mathrm{V}$ and $\mathrm{Cu}$. The emphasis lies \\ on the indirect exchange interaction of the magnetic sandwiches across the \\ antiferromagnetic or non-magnetic spacers, and on the profiles of the intrin- \\ sic resp. induced magnetic moments. Among other results we find (i) that \\ $\mathrm{Ni}$ is very sensitive on its neighborhood and that (ii) at the interface to $\mathrm{Fe}$, \\ $\mathrm{V}$ gets almost as strongly polarized as $\mathrm{Cr}$, whereas in the interior layers, the \\ $\mathrm{V}$ sandwich remains non-polarized.
}

PACS numbers: 75.70.-i, 75.70.Cn

\section{Introduction}

The exchange coupling between ferromagnetic metal sandwiches across non-magnetic and antiferromagnetic spacers is a known interesting phenomenon, which is understood by various theories using, e.g. the different reflection and interference properties of electronic waves of different spin polarization in the systems considered, e.g. $[1,2]$. Furthermore, it is well known that the above-mentioned coupling depends in an oscillatory way not only on a thickness of the spacer, but also on thicknesses of the magnetic layers of the system $[3,4]$, which offers additional possibilities to tune the magnetic properties of the system. Finally, all these properties are interesting with respect to possible applications, e.g. by using such systems as magneto-resistive reading-heads in magnetic storage devices, since, e.g. the resistance strongly depends on the mutual orientation of the magnetization of the ferromagnetic layers, which is known as the GMR-effect, see e.g. [5].

To cope with the rapidly extending experimental possibilities to study ternary and even more complex systems by using, e.g. double-wedge techniques [6], we have extended our, ab-initio calculations to complex binary and ternary systems with

*Corresponding author 
up to 20 non-equivalent layers. Such $a b$-initio calculations have been already used successfully by various groups, e.g. $[7,8,4]$ and although they typically overestimate the amplitude of the thickness oscillations of the exchange interaction by one order of magnitude compared to experiments, the wavelengths and phases are reliably calculated, as shown, e.g. by a comparison, performed in Ref. [4], of the results from Ref. [7] with the results obtained by the present method.

\section{Method and results}

As in Ref. [4], we use the first-principles LMTO package of Anderson and Jepsen $^{\dagger}$, in its non-relativistic version and in the atomic-sphere approximation but with all important corrections, by which (i) the accuracy of the $k$-space integrals is enhanced and (ii) the overlapping and empty parts of the elementary Wigner-Seitz-cell and - at the same time - higher angular momenta are taken into account. In the simplest case, the systems considered are of the kind $\left(n_{1} M_{1} / n_{2} M_{2}\right)_{\infty}$, where $M_{1}$ and $M_{2}$ are the magnetic or spacer metals, respectively, while the $n_{i}$ denote the numbers of respective layers, and $(\ldots)_{\infty}$ reminds to the periodic continuation of the antiferromagnetic unit cell, which comprises $2\left(n_{1}+n_{2}\right)$ atoms, in all three directions. The interface orientations considered are $(0,0,1)$ and $(0,1,1)$ but below we only present results for the $(0,0,1)$ case. The exchange interaction $J$ is obtained from the energy difference $\Delta E=E_{\uparrow, \downarrow}-E_{\uparrow, \uparrow}$ for the antiferromagnetic and the ferromagnetic orientation of this unit cell by the equation $J=\Delta E /(4 A)$, where $A$ is the area of the unit cell. Thus $\Delta E>0$ means ferromagnetic coupling.

In Ref. [9] we have already studied the influence of $\mathrm{Ni}$ replacing Co at the interfaces of $\left(\mathrm{Co}_{4} / \mathrm{Cu}_{n}\right)_{\infty}$ multilayers and found a weakening of the interaction by almost a factor $c a .0 .25$, and a phase shift of the $\Delta E$ vs. $n$ oscillations by almost one monolayer to the right for the corresponding $\left(\mathrm{Ni}-\mathrm{Co}_{2} \mathrm{Ni} / \mathrm{Cu}_{n}\right)$ systems. Here we find that also the magnetization of the $\mathrm{Ni}$ layers is drastically influenced by its position with respect to the interface. A typical example is shown in Fig. 1a. The Ni moment is strongly reduced at the interface, i.e. from the bulk value 0.6 to about $0.4 \mu_{\mathrm{B}}$, whereas in the central position of a $\mathrm{Ni}_{5}$ sandwich one obtains a $10 \%$ enhancement, and for the $\mathrm{Ni}_{7}$ sandwiches even an oscillatory behavior, with enhanced values at layers 3 and 5 , but a reduction almost to the bulk value at the central layer 4 . Another interesting observation is a significant induced polarization, $c a$. $-0.015 \mu_{\mathrm{B}}$, of the $\mathrm{Cu}$ layers at the interface. Interestingly, this $\mathrm{Cu}$ polarization is antiparallel to that of $\mathrm{Ni}$, whereas it would be parallel to $\mathrm{Co}$ at the corresponding $\mathrm{Co} / \mathrm{Cu}$ interface, as we have found in Ref. [10]. Furthermore, we find for a ternary system $\left(\mathrm{NiCo}_{n} \mathrm{NiCu}_{2}\right)_{\infty}$ in Fig. $1 \mathrm{~b}$ that the induced $\mathrm{Cu}$ moments mainly see the neighboring $\mathrm{Ni}$ layer and not the Co layers beyond. Finally we found from the systems with $n \geq 4 \mathrm{Cu}$ layers that the induced Cu polarization is typically reduced by a factor of about 0.3 at the 2 nd $\mathrm{Cu}$ layer near the interface, with respect to that of the 1st layer, i.e. there is again a significant polarization, and no change of sign, in layer 2. This should be contrasted to the situation with a Co/Cu interface, where - as already mentioned - the 1 st $\mathrm{Cu}$ layer at the interface is polarized

tWe thank O.K. Andersen and O. Jepsen for their LMTO programs. 

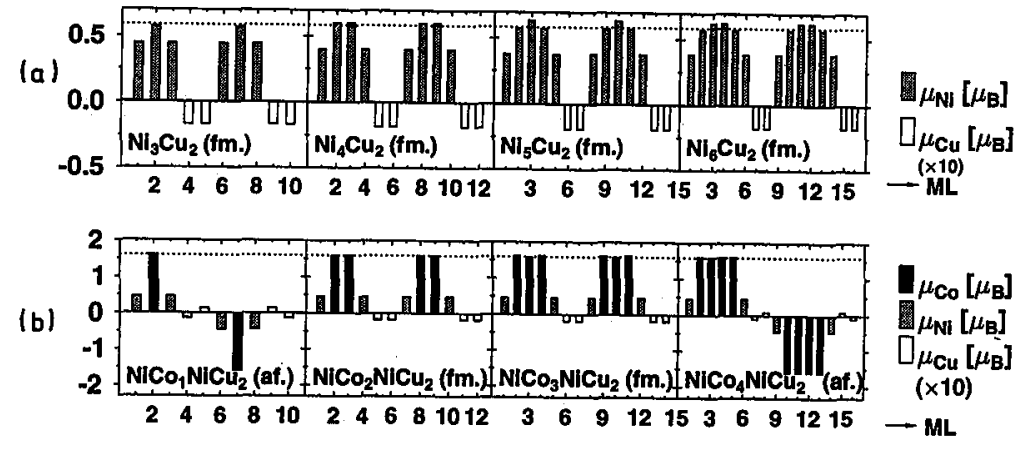

Fig. 1. Magnetization profiles in $\mathrm{Ni} / \mathrm{Cu}$ (a) and $\mathrm{Co}-\mathrm{Ni} / \mathrm{Co}$ (b) multilayers.

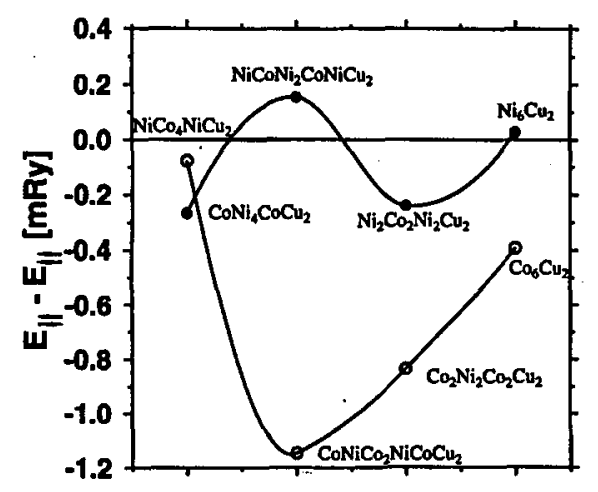

Fig. 2. Exchange interaction in $\left(\mathrm{Ni}-\mathrm{Co}_{4}-\mathrm{Ni} / \mathrm{Cu}_{2}\right)$ and related multilayers.

antiparallel to $\mathrm{Co}\left(\mu \approx-0.01 \mu_{\mathrm{B}}\right)$, whereas the 2 nd layer must be polarized parallel to $\mathrm{Co}\left(\mu \approx+0.005 \mu_{\mathrm{B}}\right)$, see [10].

In any case it should be noted that (i) such small induced $\mathrm{Cu}$ moments of the order of $0.01 \mu_{\mathrm{B}}$ can be measured by X-ray circular dichroism [11], and (ii) that, according to our results, $\mathrm{Cu}$ layers, which are more than two monolayers away from the interface, are practically unpolarized.

Figure 2 shows that the exchange interaction between the magnetic slabs in ternary multilayers of $\mathrm{Co}, \mathrm{Ni}$ and $\mathrm{Cu}$ changes drastically with the details of the composition and the position of the various layers. E.g. for the Co-based multilayers of the lower curve in Fig. 2, $\left(\mathrm{Ni}-\mathrm{Co}_{4}-\mathrm{Ni} / \mathrm{Cu}_{2}\right)_{\infty},\left(\mathrm{Co}-\mathrm{Ni}-\mathrm{Co}_{2}-\mathrm{Ni}-\mathrm{Co} / \mathrm{Cu}_{2}\right)_{\infty}$, $\left(\mathrm{Co}_{2}-\mathrm{Ni}_{2}-\mathrm{Co}_{2} / \mathrm{Cu}_{2}\right)_{\infty},\left(\mathrm{Co}_{6} / \mathrm{Cu}_{2}\right)_{\infty}$, the exchange between the magnetic slabs is antiferromagnetic, i.e. $\Delta E<0$ : For the first system, with one Ni layer at the interface, it is rather weak, but it becomes very strong when the Ni layer moves into the interior of the Co sandwich, i.e. for the 2nd and 3rd point of the lower curve. In contrast, for the Ni-based multilayers of the upper curves, the exchange alternates from antiferromagnetic $(\Delta E<0)$ to ferromagnetic sign $(\Delta E>0)$ and vice versa in the sequence $\left(\mathrm{Co}-\mathrm{Ni}_{4}-\mathrm{Co} / \mathrm{Cu}_{2}\right)_{\infty},\left(\mathrm{Ni}-\mathrm{Co}-\mathrm{Ni}_{2}-\mathrm{Ci}-\mathrm{Ni} / \mathrm{Cu}_{2}\right)_{\infty}$, 

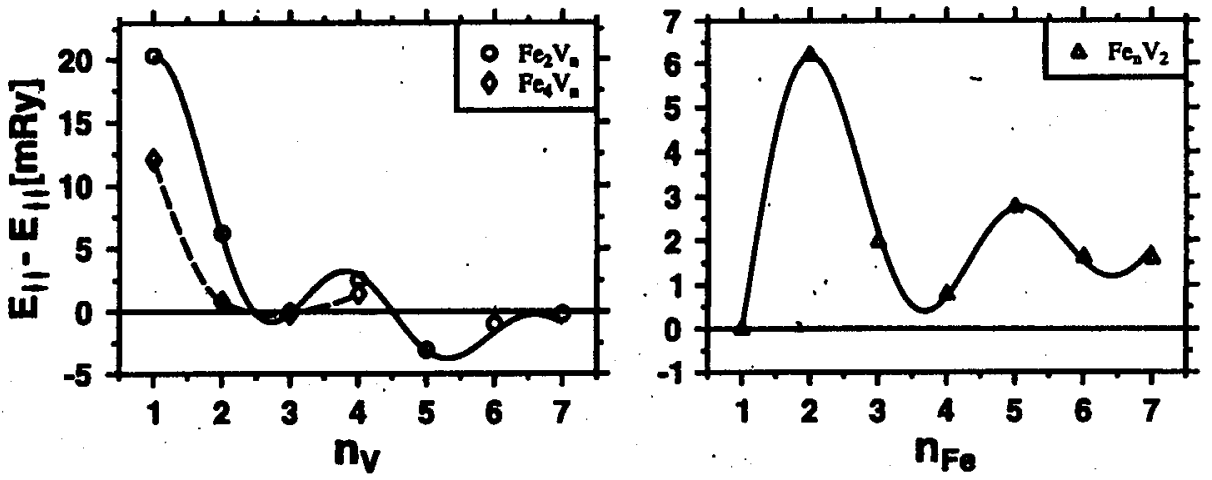

Fig. 3. Exchange interaction in $\left(\mathrm{Fe}_{2} / \mathrm{V}_{n}\right)$ and $\left(\mathrm{Fe}_{n} / \mathrm{V}_{2}\right)$ multilayers.

$\left(\mathrm{Ni}_{2}-\mathrm{Co}_{2}-\mathrm{Ni}_{2} / \mathrm{Cu}_{2}\right)_{\infty},\left(\mathrm{Ni}_{6} / \mathrm{Cu}_{2}\right)_{\infty}$. Generally speaking, the magnitude of the exchange is stronger when $\mathrm{Co}$, and not $\mathrm{Ni}$, is at the interface.

Finally we have studied $\left(\mathrm{Fe}_{n} / \mathrm{V}_{m}\right)_{\infty}$-multilayers. This system is interesting due to the following reason: $V$ would be nonmagnetic as a bulk metal. However, in the above-mentioned multilayers we have found that the first $\mathrm{V}$ layer at the (001)-interface is strongly polarized, $\mu=-0.6 \mu_{\mathrm{B}}$, antiparallel to Fe. But in contrast to $\mathrm{Cr}$, all other $\mathrm{V}$ layers are non-polarized. Also the exchange interaction $J$ of such systems shows strong oscillations as a function of $n$ and $m$, see Fig. 3 .

\section{Acknowledgments}

This work has been carried out under the bilateral project DFG/PAN 436 POL and the Committee for Scientific Research grant 2P 30200507 (SK). Our thanks go also to the Munich, Poznan and Regensburg Computer Centers.

\section{References}

[1] D.M. Edwards, J. Mathon, R.B. Muniz, M.S. Phan, Phys. Rev. Lett. 67, 493 (1991).

[2] P. Bruno, Phys. Rev. B 52, 411 (1995).

[3] S.S.P. Parkin, N. More, K.P. Roche, Phys. Rev. Lett. 64, 2304 (1990).

[4] S. Krompiewski, F. Süss, U. Krey, Europhys. Lett. 26, 303 (1994).

[5] M.N. Baibich, J.M. Broto, A. Fert, F.N. Van Dau, F. Petroff, P. Etienne, G. Creuzet, A. Friedel, J. Chazelas, Phys. Rev. Lett. 61, 2472 (1988).

[6] P.J.H. Bloemen, M.T. van de Vorst, M.T. Johnson, R. Coehoorn, W.J.M. de Jonge, Mod. Phys. Lett. B 9, 1 (1994).

[7] F. Herman, J. Sticht, M. van Schilfgaarde, J. Appl. Phys. 69, 4783 (1991).

[8] P. Lang, L. Nordström, R. Zeller, P.H. Dederichs, Phys. Rev. B 50, 13058 (1994).

[9] S. Krompiewski, F. Süss, U. Krey, J. Magn. Magn. Mater. 149, L251 (1995).

[10] S. Krompiewski, F. Süss, B. Zellermann, U. Krey, J. Magn. Magn. Mater. 148, 198 (1995).

[11] G. Schütz, M. Knülle, H. Ebert, Phys. Scr. Vol. T 49, 302 (1993). 